\title{
First experience of cadaveric renal transplantation in Sri Lanka
}

\author{
P K Harrischandra ${ }^{1}$, D N Jayatunge ${ }^{1}$, U B Rambukwella ${ }^{1}$, I B Sumithraarachchi ${ }^{1}$, U I Karunadasa ${ }^{2}$, \\ A S Abeyagunawardena ${ }^{2}$
}

(Index words: renal transplantation, delayed graft function, primary non function, graft survival, and acute rejection)

\begin{abstract}
Objectives To analyse the outcome of 50 consecutive cadaveric renal transplants performed in Sri Lanka.

Methods This was a single-centre longitudinal cohort study conducted in the Nephrology and Kidney Transplant Unit, Teaching Hospital Kandy, Sri Lanka. Records of 50 Sri Lankan cadaveric renal transplant recipients from 7th December 2004 to 1st September 2013 were reviewed and categorized according to early graft function, cold ischaemia time and the duration of dialysis before surgery.

Results Out of the 50 patients, twenty one (42\%) had immediate graft function (IGF), 26 (52\%) delayed graft function (DGF), and 2 (4\%) primary non function (PNF). The average cold ischaemia time (CIT) was 8.8 hours. Out of 23 patients who died during the study period, 19 (82.6\%) died although they had a functioning graft. Infection was the commonest cause of death $(n=15$; $65.2 \%)$. Rejection occurred in four (17.4\%). Patient survival was $77.3 \%$ at one year, $63.0 \%$ at three years and $46 \%$ at five years. Graft survival was $93.2 \%$ at one year, $88.9 \%$ at 3 years and $84.6 \%$ at 5 years when death with a functioning graft was censored. Univariate analysis revealed that neither CIT nor duration of dialysis before transplant affected patient survival. There was no significant difference in patient survival between DGF and IGF.

Conclusions The events occurring in the early period following renal transplantation do not have a significant impact on long term graft outcome or patient survival. Infection is the commonest cause of death after renal transplantation and efforts should be directed to understand and prevent this complication.
\end{abstract}

Ceylon Medical Journal 2017; 62: 83-86

DOI: http://doi.org/10.4038/cmj.v62i2.8471

\section{Introduction}

Renal transplantation offers superior life expectancy and quality of life, compared to dialysis, in patients with end-stage renal disease (ESRD). However, the initial risks of mortality and morbidity are high [1]. During the first postoperative year the risks of death for both transplantation and dialysis are not significantly different [2].

Yet, long-term survival of patients who undergo transplantation is significantly better irrespective of their primary renal disease when compared to patients who are listed but remain on dialysis. Successful renal transplantation triples the life expectancy of a listed renal failure patient [2].

The main limiting factor for renal transplantation is the unavailability of donor kidneys. In this context, cadaveric renal transplantation at present seems to offer a more practical solution [3].

Factors such as age of the donor and recipient, cold ischemic time and cause of donor's death affect the outcome of primary cadaveric renal transplantation [4,5]. United Kingdom has a policy of exchanging kidneys among centres on the basis of HLA matching, and it is fully justified especially when there is a 0:0:0 mismatch for HLA between donor and recipient, highlighting the strong importance of HLA matching [6].

Causes of graft loss following transplantation after the first year include death with functioning transplants, chronic rejection, fibrosis and glomerular pathologies [7,8]. The principal causes of death in renal transplant recipients with a functioning graft are cardiovascular disease (CVD), infection, and malignancy [9-11]. In a study by Woo et al the actuarial graft survival of cadaveric transplant patients was $84 \%$ at one year, $68 \%$ at five years and 55\% at ten years, when death with a functioning graft was censored. This is calculated from the date of transplantation to the date of irreversible graft failure. When deaths with a functioning graft were counted as graft loss, graft survival

${ }^{1}$ Nephrology and Kidney Transplant Unit, Teaching Hospital Kandy and ${ }^{2}$ Department of Paediatrics, Faculty of Medicine, University of Peradeniya, Sri Lanka.

Correspondence: ASA, e-mail: <asiriabey26@gmail.com>. Received 19 November 2016 and revised version accepted 21 March 2017.

This is an open-access article distributed under the terms of the Creative Commons Attribution License, which permits unrestricted use, distribution, and reproduction in any medium, provided the original author and source are credited. 
was $81 \%$ at one year, $60 \%$ at five years, and $45 \%$ at ten years $[12,13]$.

Patient survival figures of Sri Lankan cadaveric renal transplant patients have not been studied previously. Since the number of patients living with ESRD is increasing in our country the demand for renal grafts is also increasing. Cadaveric renal transplantation is therefore being established as a viable supplement to live donor transplantation.

In this paper we present our experience in cadaveric renal transplantation in a single centre in Sri Lanka.

\section{Methods}

Data for our study was taken from 50 consecutive cadaveric renal transplants done at the Nephrology and Kidney Transplant Unit, Teaching Hospital Kandy between 7th December 2004 and 1st September 2013. Kidney transplant patients are regularly followed up on a monthly basis at Transplant Nephrology unit at Teaching Hospital Kandy. Seven patients who were transferred to other renal units subsequently were also included. Patients were followed up until either death or 1 September 2013, whichever occurred first.

Follow up assessment included renal function tests, blood pressure measurement and urine analysis with appropriate drug level estimation. All patients received standard triple immunosuppression therapy with tacrolimus, mycohenolate mofetil and prednisolone.

We assessed current renal profile, renal imaging and quality of life of the patients. Data were collected from the clinical records, operation details and discharge summaries in the immediate postoperative period. Additional information was obtained by contacting the patients or their immediate family. Living patients were interviewed when they came for clinic follow up.

The quality of life was assessed using the SF36 questionnaire. Here 8 health related parameters were assessed where the maximum score of 100 means no disability and zero is equivalent to highest disability. Scores were calibrated so that 50 was the average score or norm.

We analysed two survival outcomes, patient and graft survival. Patient survival period was defined as the duration from date of transplant until death. Graft failure was defined as return to dialysis; death with functioning graft was counted as lost to follow up.

Patients who did not require dialysis following transplantation were categorised as having immediate graft function (IGF). Patients who needed one or more haemodialysis sessions following transplantation prior to the onset of graft function were categorised as having delayed graft function (DGF). Patients whose graft never functioned were categorised as having primary non function (PNF).
Ethical approval was obtained from the Scientific and Ethics Committee, Teaching Hospital Kandy. All participants gave informed consent.

\section{Statistical analysis}

Data was analysed using SPSS software version 17. Kaplan-Meier survival curves were computed according to graft function, cold ischaemic time (CIT), duration of dialysis before surgery and serum creatinine level at discharge. The log-rank test was used to assess the significance of differences in survival. Chi-square test was used to compare differences in frequency of events in different groups.

\section{Results}

Fifty patients were included in the analysis. Thirty five (70\%) were males and 15 were females. Median follow up was 3.5 years. Mean age at transplant was 40.5 years. Mean duration of dialysis before surgery was 21.2 months. The mean CIT was 8.8 hours. Twenty one (42\%) had IGF, 26 (52\%) had DGF and two (4\%) had PNF.

Twenty three patients died during the study period. Nineteen (38\%) died with a functioning graft, and four (8\%) died after their grafts had failed. Infection, which occurred in 15 (30\%), was the commonest cause of death. Rejection occurred in four (8\%). Two patients (4\%) died due to cardiovascular causes. One patient committed suicide and another died due to a perforated gastric ulcer. Of the patients who died of infections, 10 died of pneumonia and one each died of cytomegalovirus infection, fungal infections of the brain, chicken pox, sepsis and tuberculosis.

Patient survival of total population was $77.3 \%$ at one year, $63.0 \%$ at three years, and $46 \%$ at five years. When PNF was included in the survival analysis there was a significant difference between the three groups $(p<0.001)$. But when comparing only the IGF and DGF groups there was no significant difference in the survival ( $p=0.512$ ). Univariate analysis of patient survival in patients divided by early graft function, and survival with regard to cold ischemia time is shown in Figures 1 and 2 respectively.

There was no significant difference in survival according to serum creatinine levels $(<200 \mu \mathrm{mol}, 200-400$ $\mu \mathrm{mol}$ and $>400 \mu \mathrm{mol})$ at the time of discharge $(p=0.103)$. There was no significant difference in patient survival according to duration of dialysis before transplant $(<24$ months and $>24$ months) $(p=0.270)$ or CIT ( $<4$ hrs, $4-8$ hrs and $>8$ hrs $)(p=0.133)$.

Graft survival for the total population was $93.2 \%$ at one year, $88.9 \%$ at 3 years and $84.6 \%$ at 5 years when death with a functioning graft was censored. Out of the 50 patients who underwent cadaveric transplants, only 4 patients developed graft rejection and all four had died within 3 months of surgery. 


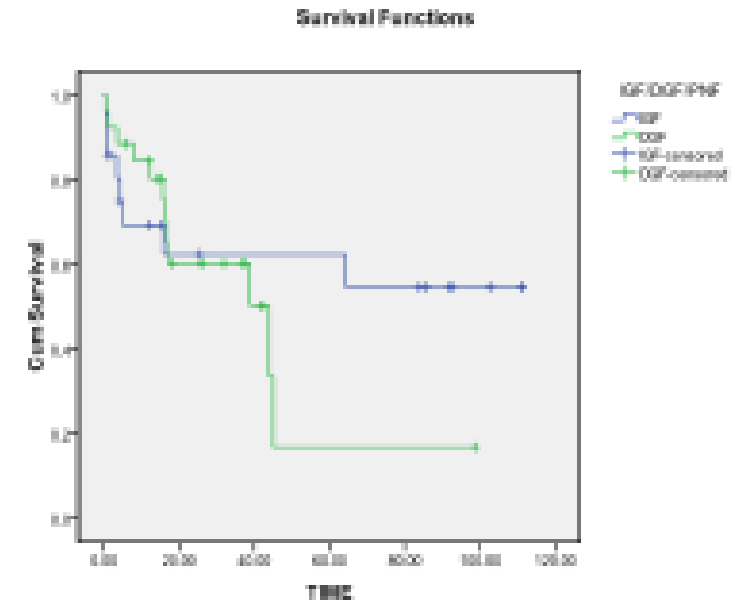

Figure 1. Patient survival by early graft function.

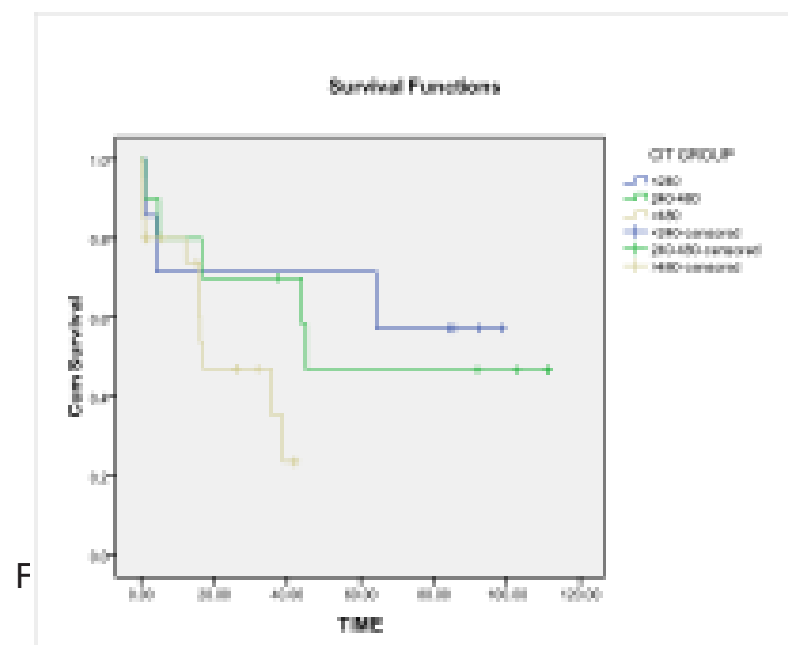

Table 1. Renal biopsy findings

\begin{tabular}{lcc}
\hline & Frequency in years & Percentage \\
\hline No rejection & 18 & 46.2 \\
One episode & 17 & 43.6 \\
Two episodes & 4 & 10.3 \\
Total & 39 & 100.0 \\
\hline
\end{tabular}

The majority of the living patients (73.3\%) had normal creatinine (less than $150 \mu \mathrm{mol}$ ) levels. There was no significant association between serum creatinine values and urine output on discharge $(p=0.061)$ or at the time of study $(p=0.219)$.

The number of patients with rejection is shown in Table 1. All acute rejection episodes were confirmed by biopsies which were read by a single pathologist. A kidney biopsy was performed if a patient developed a $\geq 25 \%$ rise in the baseline serum creatinine level and reduced urine output. No protocol biopsies were performed. There was no significant difference in survival according to the number of episodes of rejection $(p=0.183)$.
The SF 36 health survey results were available for 25 living patients. The mean of the physical component was 53.4 and mean of the mental component was 50.2. Only one patient complained of a problematic arteriovenous fistula (AVF) site. We were able to ligate and excise the AVF in that patient. Three people had erectile dysfunction and apart from them none complained of lack of sexual drive.

\section{Discussion}

This study analysed survival and factors associated with graft survival in patients who received cadaveric transplants at the Nephrology and Transplant Unit at Teaching Hospital Kandy. Although it is from a single centre, it is one of the largest single centre reports on long-term graft outcome in cadaveric transplant patients in Sri Lanka.

Woo et al reported that majority of cadaveric transplant recipients died due to CVD [12]. Risk factors for CVD include hypertension, dyslipidemia, posttransplant diabetes, inflammation and anaemia [14-18]. The developed world has overcome the cyclosporine era where infections and malignancies were the common cause of death [12]. Since our major cause of death was infections (65.2\%) it seems likely that we have not passed that stage.

In our present study patient survival at 1 year was $77.3 \%$ and death censored graft survival was $93.2 \%$. Many studies have shown that graft loss is associated with an increased risk of death. In our present series, four patients died due to primary graft failure. None of our living patients suffered from graft loss. Out of 39 patients, 10 did not undergo renal biopsy and in the 29 who underwent biopsy 8 did not show any rejection. The possible reason for lesser number of rejection episodes may be because our patients had not lived long enough for graft loss to occur.

Rodrigo et al showed a difference in the acute rejection rate between IGF and DGF. DGF was higher than IGF (42\% vs 21\%) [19]. They also showed a superior graft survival in patients with IGF compared to DGF.

Even though acute rejection and poor early graft function are recognized predictors of poor graft survival, in our study we failed to show an association with these predictors and patient survival. The reason for this lack of association in our study is unclear. We were also unable to show an association with the length of pretransplant dialysis and patient outcome.

The death censored graft survival rates in our study were comparable to those in other series of cadaveric transplants. However, our patient survival rates were relatively low and this fact cannot be ignored. Infection was the commonest cause of death. During the first year after transplantation where immunosuppression is most intense, renal patients face a threat of infections [20]. Conventional immunosuppression was used with basiliximab and methylprednisolone at induction, along 
with tacrolimus/cyclosporine, mycophenolate mofetil and prednisolone based triple immunosuppression as maintenance. The relatively lower patient survival rates due to infection cannot therefore be attributed to excessive immunosuppression. The management of infections in transplant recipients needs to be improved. This includes preventive strategies, early identification and treatment which we believe would greatly improve the outcome of cadaveric transplants [21].

A study by Schnitzler et al highlights the importance of HLA-based exchanging of kidneys is cost saving [22]. In our series we were unable to perform HLA matching due to economic constraints, but hope that HLA matching prior to cadaveric transplantation will become a reality for us in the near future. This will undoubtedly improve both graft and patient survival.

In Sri Lanka cadaveric renal transplantation is still in its infancy. However, we believe that with the high incidence of ESRD in the country, brought about by the epidemic of chronic kidney disease of unknown origin, this provides a viable solution to the scarcity of living donors. Though patient survival was a problem in our series we believe that the ultimate outcome was greatly improved when considering the poor survival rates in haemodialysis in resource-poor settings. No doubt the programme needs to improve with better dialysis, HLA matching, better retrieval strategies, better management of infections etc. We hope that this study will highlight our cadaveric transplant programme and lead to implementation of a national programme.

In conclusion this study shows that events occurring in the early period following renal transplantation are not associated with long-term graft outcome or patient survival. Infections were the commonest cause of death following renal transplantation and efforts should be directed to understand and prevent this complication.

\section{Acknowledgement}

We thank Dr.T Abeysekara and Dr. AWM Wazeel at Teaching Hospital Kandy for their assistance in followingup the patients during this study.

\section{Conflicts of interest}

There are no conflicts of interest.

\section{References}

1. Jassal SV, Krahn MD, Naglie G, et al. Kidney Transplantation in the Elderly: A Decision Analysis. J Am Soc Nephrol 2003; 14: $187-96$

2. Oniscu GC, Brown H, Forsy. The J. Impact of Cadaveric Renal Transplantation on Survival in Patients Listed for Transplantation. J Am Soc Nephrol 2005; 16: 1859-65.

3. Smith PK, Marshall VC, Mathew TH, et al. Cadaveric renal transplant. Lancet 1967; 290: 59-62.
4. Weiss-Salz I, Mandel M, Galai N, et al. Factors associated with primary and secondary graft failure following cadaveric kidney transplant. Clin Transplant 2004; 18: 571-5.

5. Swanson S, Hypolite I, Agodoa L, et al. Effect of Donor Factors on Early Graft Survival in Adult Cadaveric Renal Transplantation. Am J Transplant 2002; 2: 68-75.

6. Morris PJ, Johnson RJ, Fuggle SV, et al. Analysis of factors that affect outcome of primary cadaveric renal transplantation in the UK. Lancet 1999; 354: 1147-52.

7. El-Zoghby Z, Stegall M, Lager D, et al. Identifying Specific Causes of Kidney Allograft Loss. American Journal of Transplantation 2009; 9: 527-35.

8. Morris PJ. Transplantation in the 21st century. Kidney Int 1997; 51: S51-S5.

9. Raine AEG. Cardiovascular complications after renal transplantation. In: Morris PJ, eds. Kidney Transplantation: Principles and practice. 4th ed. Philadelphia: WB Saunders, 1994: 39-341.

10. Mallick NP, Jones E, Selwood. The European (European Dialysis and Transplantation Association-European Renal Association) Registry. Am J Kidney Dis 1995; 25: 176-87.

11. Disney APS. Demography and survival of patients receiving treatment for chronic renal failure in Australia and New Zealand: Report on dialysis and renal transplantation treatment from the Australian and New Zealand Dialysis and transplant registry. Am J Kidney Dis1995; 25: 165-75.

12. Woo YM, Jardine AG, Clark AF, et al. Early graft function and patient survival following cadaveric renal transplantation. Kidney Int 1999; 55: 692-9.

13. Expert Group on Renal Transplantation. European best practice guidelines for renal transplantation. Section IV: Longterm management of the transplant recipient. NephrolDial Transplant 2002; 17: 60-7.

14. Mangray M, Vella J, Hypertension After Kidney Transplant. Am J Kidney Dis 2011; 57: 331-41.

15. Lentine KL, Brennan DC, Schnitzler MA. Incidence and predictors of myocardial infarction after kidney transplantation. J Am Soc Nephrol 2005; 16: 496-506.

16. Hjelmesaeth J, Hartmann A, Leivestad T, et al. The impact of early-diagnosed new-onset post-transplantation diabetes mellitus on survival and major cardiac events. Kidney Int. 2006; 69: 588-95.

17. Neale J, Smith A. Cardiovascular risk factors following renal transplant. World J Transplant 2015; 5: 183-95.

18. Rigatto C, Foley R, Jeffery J, Negrijn C, Tribula C, Parfrey P. Electrocardiographic Left Ventricular Hypertrophy in Renal Transplant Recipients: Prognostic Value and Impact of Blood Pressure and Anemia. J Am SocNephrol 2003; 14: 462-8.

19. Rodrigo E, Fernanddez-Fresnedo G, Ruiz JC,et al. Similar impact of slow and delayed graft function on renal allograft outcome and function. Transplant Proc 2005; 37: 1431-2. Fishman J. Infection in Renal Transplant Recipients. Semin Nephrol 2007; 27: 445-61.

20. Jha V. Post-transplant infections: An ounce of prevention. Indian J Nephrol 2010; 20: 171-8.

21. Schnitzler MA, Hollenbeak C, Cohen D, et al. The economic implication of HLA matching in cadaveric renal transplantation. N Engl J Med 1999; 341: 1440-6. 